\title{
Rhythmic Keccak: SCA Security and Low Latency in HW
}

\author{
Victor Arribas ${ }^{1}$, Begül Bilgin ${ }^{1}$, George Petrides ${ }^{2}$, \\ Svetla Nikova ${ }^{1}$ and Vincent Rijmen ${ }^{1}$ \\ 1 KU Leuven, imec-COSIC, Belgium, name.surname@esat.kuleuven.be \\ ${ }^{2}$ Vrije Universiteit Brussel, Belgium, george.petrides@vub. be
}

\begin{abstract}
Glitches entail a great issue when securing a cryptographic implementation in hardware. Several masking schemes have been proposed in the literature that provide security even in the presence of glitches. The key property that allows this protection was introduced in threshold implementations as non-completeness. We address crucial points to ensure the right compliance of this property especially for low-latency implementations. Specifically, we first discuss the existence of a flaw in DSD 2017 implementation of KECCAK by Gross et al. in violation of the noncompleteness property and propose a solution. We perform a side-channel evaluation on the first-order and second-order implementations of the proposed design where no leakage is detected with up to 55 million traces. Then, we present a method to ensure a non-complete scheme of an unrolled implementation applicable to any order of security or algebraic degree of the shared function. By using this method we design a two-rounds unrolled first-order KECCAK- $f$ [200] implementation that completes an encryption in $20.61 \mathrm{~ns}$, the fastest SCA secure implementation in the literature to this date.
\end{abstract}

Keywords: Glitch · non-completeness · threshold implementation · consolidated masking scheme $\cdot$ domain-oriented masking

\section{Introduction}

Physical attacks are a serious threat to cryptographic implementations, capable of retrieving important information such as the secret key. In particular, Side-Channel Attacks (SCA), which are based on observing the behavior of the device without making any changes on it or its working conditions, are used frequently due to their relatively low cost and difficulty to be detected. In this paper, we use Differential Power Analysis (DPA), which exploits the relation between the intermediate values produced internally during the calculations and the instantaneous power consumption of the cryptographic device [KJJ99]. However, our observations can be generalized to other forms of SCA, such as the ones exploiting the electromagnetic emanation of the device [GMO01].

Different countermeasures have been proposed that aim to make the power consumption of a cryptographic device independent of the intermediate values. Here, we focus on Threshold Implementations (TI) [NRR06, NRS08, NRS11] and Domain Oriented Masking (DOM) [GMK16], which are based on secret sharing schemes and techniques from MultiParty Computation (MPC). Moreover, they have the advantage of providing theoretical security on hardware if implemented according to the non-completeness property defined in $\left[\mathrm{NRR} 06, \mathrm{BGN}^{+} 14 \mathrm{a}\right]$, if fed with enough entropy and if the device works under the independent leakage assumption as described in [DFS15]. There are several papers in the literature applying these countermeasures on the KECCAK permutations: Bertoni et 
al. [BDPA10a] and Bilgin et al. $\left[\mathrm{BDN}^{+} 14\right]$ with first-order resistant implementations and more recent work by Gross et al. [GSM17a] that proposes higher-order countermeasures.

Our contribution. In this paper, we first briefly summarize KECCAK's sponge function, and TI and DOM schemes (Sect. 2). Then, we analyze the recently published higher-order DOM KECCAK implementations [GSM17a] and point out a flaw that can possibly lead to successful attacks. We describe how careful tracing of the non-completeness property can be done to fix this flaw for any order. We show the different behavior between the original design and our proposal for first- and second-order implementations using TVLA with 55 million of traces (Sect. 3). Finally, we discuss how TI can be used for unrolled implementations without breaking the non-completeness property for the first time in the literature. We present a first-order secure low latency KECCAK implementation that performs an encryption in 20.61ns making it the fastest SCA secure implementation published to date (Sect. 5).

\section{Preliminaries}

\section{$2.1 \quad$ Keccak Permutations}

KECCAK is a family of sponge functions using the permutations KECCAK- $f[b]$ where $b \in$ $\{25,50,100,200,400,800,1600\}$ defines different state sizes [BDPA10b]. KECCAK [BDPA10b] is the NIST SHA-3 standard [NIS15]. Among other properties, KECCAK stands out due to its high performance when implemented in hardware and its great area/speed trade-offs. The security claims of these variants follow the Matryoshka principle, i.e. analysis of KECCAK with small sizes can easily be linked to the variants with bigger sizes. Benefiting from this property, we work on KECCAK- $f$ [200], which is suitable for lightweight architectures, and note that the concepts presented in this paper can naturally be extended to other choices of $b$. Moreover, since we simply work on KECCAK- $f$ this work also covers KETJE $\left[\mathrm{BDP}^{+} 16 \mathrm{a}\right]$ and KEYAK $\left[\mathrm{BDP}^{+} 16 \mathrm{~b}\right]$ authenticated encryptions.

KECCAK- $f$ operates on a three-dimensional state $S$, where the bit in the coordinate $(x, y, z)$ is denoted by $S[x, y, z]$. A round consists of five steps:

$$
\begin{aligned}
& R=\iota \circ \chi \circ \pi \circ \rho \circ \theta \text {, with } \\
& \theta: \quad S[x, y, z] \quad \leftarrow S[x, y, z] \oplus \bigoplus_{y^{\prime}=0}^{4} S\left[x-1, y^{\prime}, z\right] \oplus \bigoplus_{y^{\prime}=0}^{4} S\left[x+1, y^{\prime}, z-1\right], \\
& \rho: \quad S[x, y, z] \quad \leftarrow S[x, y, z-(t+1)(t+2) / 2], \\
& \text { with } t \text { satisfying } 0 \leq t<24 \text { and }\left(\begin{array}{ll}
0 & 1 \\
2 & 3
\end{array}\right)^{t}\left(\begin{array}{l}
1 \\
0
\end{array}\right)=\left(\begin{array}{l}
x \\
y
\end{array}\right) \text { in } \operatorname{GF}(5)^{2 \times 2} \text {, } \\
& \text { or } t=-1 \text { if } x=y=0 \text {, } \\
& \pi: \quad S[x, y] \leftarrow S\left[x^{\prime}, y^{\prime}\right] \text {, with }\left(\begin{array}{l}
x \\
y
\end{array}\right)=\left(\begin{array}{ll}
0 & 1 \\
2 & 3
\end{array}\right)\left(\begin{array}{l}
x^{\prime} \\
y^{\prime}
\end{array}\right), \\
& \chi: \quad S[x] \quad \leftarrow S[x] \oplus(S[x+1] \oplus 1) S[x+2], \\
& \iota: \quad S \quad \leftarrow S \oplus \mathrm{RC}\left[i_{r}\right] \text {, where } \mathrm{RC}\left[i_{r}\right] \text { is the round counter in cycle } i_{r} \text {. }
\end{aligned}
$$

Notice that we abuse the notation to focus on specific coordinates. Moreover, addition and subtraction in the coordinates are modular. For convenience, we provide visualizations of these round steps in App. A.1 (Fig. 8) for KeCCAK-f[200]. KeCCAK-f[200] computes 18 rounds. 


\subsection{Masking Schemes}

Different forms of masking schemes have been shown to provide provable security against Side-Channel Analysis given the independent leakage and uniform input assumption. They are based on secret sharing where each sensitive, i.e. key dependent, data $x$ is divided into $s$ pieces $\left(\mathrm{x}=\left(x_{1}, \ldots, x_{s}\right)\right)$ such that $x=x_{1} \perp \ldots \perp x_{s}$. Throughout this paper, we consider Boolean masking where $\perp$ is field addition denoted by $\oplus$ and full threshold sharing where all $s$ shares need to be combined to derive $x$. The exact value of $s$ is chosen depending on the desired security level which is specified further for different flavors of masking below.

Each function $f(x)=y$ of the cryptographic algorithm is calculated in a shared manner where $f_{i}(\mathbf{x})=y_{i}$. Masking is correct if $\bigoplus y_{i}=y$.

$\boldsymbol{d}$-Probing Model. It has been shown in [DFS15] that if a masked circuit achieves security under the $d$-probing model [ISW03] where each calculation is treated separately, independent of the timing of the circuit, then it also provides security against $d^{\text {th }}$-order Side-Shannel Analysis under the independent leakage assumption. Similar to $\left[\mathrm{RBN}^{+} 15\right.$, $\mathrm{FGP}^{+} 17$, we assume that an adversary probing a wire has information about all the intermediate values starting from the registers to the probed point of calculation. Note that this probing model is used specifically on hardware where glitches might occur in the circuit. As a result a $d$-probing adversary would acquire the knowledge of all the intermediate values used in all $d$ probed wires.

\subsubsection{Threshold Implementation (TI)}

This masking method, which is introduced by Nikova et al. [NRR06] for first-order security and extended by Bilgin et al. $\left[\mathrm{BGN}^{+} 14 \mathrm{a}\right]$ for higher-order security, is used widely on hardware since, unlike many masking schemes, it provides security under the non-ideal gate assumption. That is, the gates can glitch depending on prior inputs of that cycle before stabilizing without giving an advantage to an attacker. Below we repeat the non-completeness definition for completeness.

Definition 1 (Non-completeness $\left[\mathrm{BGN}^{+} 14 \mathrm{a}\right]$ ). The shared circuit $\mathbf{f}$ of $f$ is $d^{\text {th }}$-order non-complete if any combination of up to $d$ component functions $f_{i}$ is independent of at least one input share.

The lower bounds of number of input and output shares such that there always exists a sharing of an algebraic degree $t$ function satisfying the above non-completeness property are given in $\left[\mathrm{BGN}^{+} 14 \mathrm{a}\right]$ as follows:

$$
\begin{aligned}
s_{\text {in }} & \geq t d+1 \\
s_{\text {out }} & \geq\left(\begin{array}{c}
s_{i n_{\text {min }}} \\
t
\end{array}\right) .
\end{aligned}
$$

This bound has then been used for a variety of algorithms and security orders $\left[\mathrm{BNN}^{+} 12\right.$, $\left.\mathrm{BGN}^{+} 14 \mathrm{~b}, \mathrm{BNN}^{+} 14, \mathrm{BGN}^{+} 15, \mathrm{BDN}^{+} 14, \mathrm{CBR}^{+} 15\right]$.

We provide the sharings of an AND/XOR gate $(z=a \oplus b c)$ where $\left(d, s_{\text {in }}, s_{\text {out }}\right)=(1,3,3)$ and $\left(d, s_{\text {in }}, s_{\text {out }}\right)=(2,5,10)$ in Eqns. (3) and (10) (in App. A.2) respectively together with a graphical representation in Fig. 9 (in App. A.2). We refer to a sharing with $s_{\text {in }}=s_{\text {out }}=s$ as an $s$-sharing.

$$
\begin{aligned}
& z_{1}=a_{1} \oplus b_{1} c_{1} \oplus b_{1} c_{2} \oplus b_{2} c_{1}, \\
& z_{2}=a_{2} \oplus b_{2} c_{2} \oplus b_{2} c_{3} \oplus b_{3} c_{2}, \\
& z_{3}=a_{3} \oplus b_{3} c_{3} \oplus b_{1} c_{3} \oplus b_{3} c_{1} .
\end{aligned}
$$


In order to satisfy the non-completeness property, the compression $(\mathcal{C})$ from $s_{\text {out }}$ to $s_{\text {in }}$ shares must follow a synchronization layer such as registers. The first-order case becomes advantageous since $s_{\text {out }}=s_{\text {in }}$ and this intermediate synchronization layer is not necessary enabling a one cycle implementation. If a sharing dissatisfies the non-completeness property, we call it a complete sharing.

Another property introduced by TI is a uniform sharing. Since we only use permutations in this paper, we use the simplified definition provided below.

Definition 2 (Uniform Sharing $\left[\mathrm{BNN}^{+} 12\right]$ ). The $s$-sharing of an $n$-bit permutation $f(x)=y$ is uniform if its sharing $\mathbf{f}(\mathbf{x})=\mathbf{y}$ is an $n s$-bit permutation.

Note that this uniformity definition is compatible with the definition presented for higher-order security in $\left[\mathrm{BGN}^{+} 14 \mathrm{a}\right]$ when $\mathbf{y}$ is taken as the sharing after compression. Moreover, if this property is satisfied for each step of a cryptographic algorithm, the algorithm provides univariate security without the need of additional randomness. It has been shown in $\left[\mathrm{RBN}^{+} 15\right]$ that uniformity condition is not enough for multi-variate security in the higher-order case. The authors show that it is possible to achieve higher-order multi-variate security for TI by inserting fresh randomness to each $s_{\text {out }}$ output shares just before the compression. This refreshing $(\mathcal{R})$ naturally provides a uniform sharing.

\subsubsection{Domain Oriented Masking (DOM)}

In [GMK16], Gross et al. introduced $d^{\text {th }}$-order secure DOM-indep multiplier which uses $s_{i n}=d+1$ input shares and $d(d+1) / 2$ units of randomness. DOM multiplier assumes $s_{i n}$ domains.

The sharing structure of DOM-indep multiplier uses $d+1$ input shares for hardware and is a follow up of Reparaz et al. $\left[\mathrm{RBN}^{+} 15\right]$ which provides security given only the independence of the shared input variables. The difference between DOM-indep as opposed to $\left[\mathrm{RBN}^{+} 15\right]$ multiplier is the significant randomness optimization, from $(d+1)^{2}$ to $d(d+1) / 2$. This optimized shared multiplier has been used in [GSM17a] to provide very small implementations of KECCAK with higher-order security claim. We provide a DOM-indep AND gate $(z=a b)$ where $\left(d, s_{\text {in }}, s_{\text {out }}\right)=(1,2,4)$ and $\left(d, s_{\text {in }}, s_{\text {out }}\right)=(2,3,9)$ in Eqn. 4 below and in Eqn. 11 in appendix. The parenthesis [.] and (.) represent the mandatory and optional synchronizations respectively and $r$ refers to the randomness used for refreshing.

$$
\begin{aligned}
& z_{1}=\left(a_{1} b_{1}\right) \oplus\left[a_{1} b_{2} \oplus r\right], \\
& z_{2}=\left[a_{2} b_{1} \oplus r\right] \oplus\left(a_{2} b_{2}\right) .
\end{aligned}
$$

In [GMK16] a second multiplier called DOM-dep multiplier is also introduced. DOMdep has the advantage of not relying on the independently shared inputs assumption of other $d+1$ share schemes, such as DOM-indep and $\left[\mathrm{RBN}^{+} 15\right]$, while using less randomness. For details of DOM-dep multiplier and randomness reductions of DOM, we refer to the original paper. Here, we only focus on DOM-indep multiplier which was used in [GSM17a] and the non-completeness property. Therefore, we refer to DOM-indep as DOM for brevity.

\section{Round-Based Implementations}

KECCAK is implemented for a variety of platforms and constraints. The smallest roundbased implementation claiming security against SCA is presented in [GSM17a] and uses DOM. In this section, we first show a potentially exploitable weakness of that round based implementation based on the failure of non-completeness property. We then verify the 
observability of our claims on an FPGA using t-test based leakage detection. Finally, we discuss how a sharing using $s_{i n} \geq d+1$ shares should be implemented such that it satisfies the non-completeness property and the restrictions of such an implementation. Note that our analysis covers round-based implementations of any order but does not cover the serialized architectures presented in [GSM17a].

\subsection{Analysis of DOM-Keccak}

A traditional round-based implementation of KECCAK receives the input of $\theta$ from a register; $\theta, \rho, \pi, \chi$ and $\iota$ operations are calculated within the same clock cycle and the output of $\iota$ is written to a register. When we consider a $d+1$-share implementation, it is evident that $\chi$-refreshing needs to be separated from $\chi$-compression by registers to achieve non-completeness marking the end of the cycle. The difference between these two architectures are depicted in Figure 1.

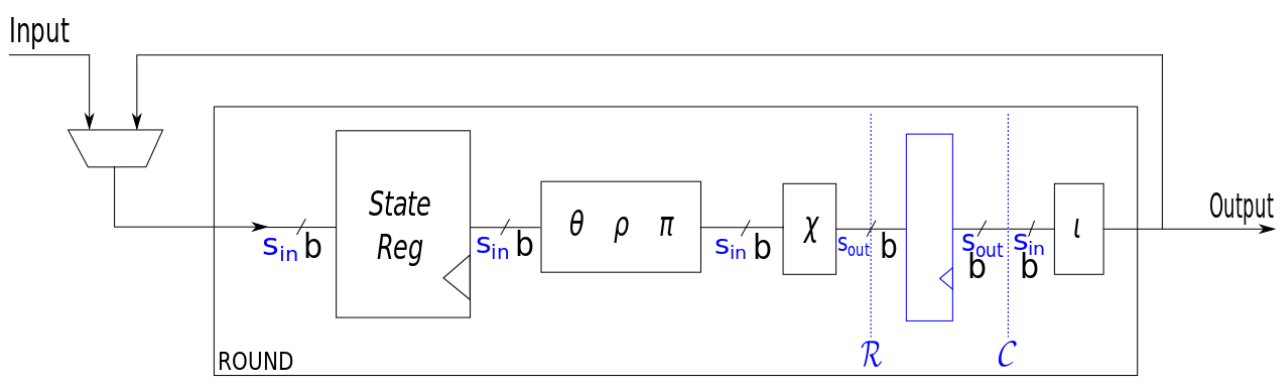

Figure 1: Datapath of protected (blue) and unprotected round-based Keccak implementation with and without $\chi$-compression respectively.

The first-order implementation in [GSM17a] uses the sharing of $\chi$ in Eqn. (5) up to the synchronization register. The randomness $r$ is taken from another (independent) part of the state. The synchronization is performed only on the cross-domain shares $S_{2}^{\prime}[x]$ and $S_{3}^{\prime}[x]$ by using a register triggered on the negative edge of the clock while triggering the state register in the positive edge (double clocking). Analyzing each linear and nonlinear step individually as is done in [GSM17a], shows that each separate step is secure against first-order SCA. Moreover, it can easily be verified that the non-completeness property is satisfied in each separate step. However, the non-completeness of any implementation should be verified from register to register since the glitchy behavior can accumulate.

$$
\begin{aligned}
& S_{1}^{\prime}[x] \leftarrow S_{1}[x] \oplus\left(S_{1}[x+1] \oplus 1\right) S_{1}[x+2] \\
& S_{2}^{\prime}[x] \leftarrow S_{1}[x+1] S_{2}[x+2] \oplus r \\
& S_{3}^{\prime}[x] \leftarrow S_{2}[x+1] S_{1}[x+2] \oplus r \\
& S_{4}^{\prime}[x] \leftarrow S_{2}[x] \oplus\left(S_{2}[x+1] \oplus 1\right) S_{2}[x+2] .
\end{aligned}
$$

After analyzing one round of the aforementioned implementation, we noticed that the non-completeness property from register to register is not satisfied for 112 out of 200 round output bits invalidating a condition for security. Below we trace back the input shares used to calculate a specific bit of the state particularizing Eqn. (1) for first-order secure implementation. A graphical representation is also provided in Fig. 10 in App. A.3.1. We use $S_{i}^{f}$ to refer to the output of $f$ for share $S_{i}$. 
Tracing back output bit $S^{\chi}[4,1,0]$ of the $\chi$ permutation:

$$
\begin{array}{rll}
\chi^{-1}: \quad S_{3}^{\chi}[4,1,0] & \leftarrow S_{2}^{\pi}[0,1,0] S_{1}^{\pi}[1,1,0] \oplus r \\
\pi^{-1}: \quad S_{2}^{\pi}[0,1,0] & \leftarrow S_{2}^{\rho}[3,0,0] \\
S_{1}^{\pi}[1,1,0] & \leftarrow S_{1}^{\rho}[4,1,0] \\
\rho^{-1}: \quad S_{2}^{\rho}[3,0,0] & \leftarrow S_{2}^{\theta}[3,0,4] \\
S_{1}^{\rho}[4,1,0] & \leftarrow S_{1}^{\theta}[4,1,4] \\
\theta_{1}^{-1}: \quad S_{2}^{\theta}[3,0,4] & \leftarrow S_{2}[3,0,4] \oplus \bigoplus_{y^{\prime}=0}^{4} S_{2}\left[2, y^{\prime}, 4\right] \oplus \bigoplus_{y^{\prime}=0}^{4} S_{2}\left[4, y^{\prime}, 3\right] \\
& & \leftarrow S_{1}[4,1,4] \oplus \bigoplus_{y^{\prime}=0}^{4} S_{1}\left[3, y^{\prime}, 4\right] \oplus \sum_{y^{\prime}=0}^{4} S_{1}\left[0, y^{\prime}, 3\right]
\end{array}
$$

From these expressions it can be derived that the output bit $S_{3}^{\chi}[4,1,0]$ of the non-linear layer depends, among others, on the input bits $S_{1}[3,0,4]$ and $S_{2}[3,0,4]$. This means that all shares of $[3,0,4]$ are used and hence, non-completeness fails. Note that similar complete output bits can also be found for higher-order implementations. We provide a second-order example in App. A.3.1.

\subsubsection{Evaluation}

To illustrate the problem presented above we tested our first-order round-based DOMKECCAK implementation that follows the structure depicted in Fig. 1 on FPGA. Note that we used our own implementation instead of the one provided by the authors of [GSM17a] in $[\mathrm{Sch} 17]^{1}$. The differences between our design and that of [Sch17] are (1) we do not use negative-edge triggering for the cross-domain shares for ease of analysis leading to a two cycle per round implementation; and (2) we always use fresh randomness to ensure that a possible problem is not caused by the degradation of uniformity.

Platform. To evaluate our design, we deploy it into a Spartan-6 Xilinx FPGA on a Sakura-G board, which is specifically designed for side-channel evaluation. To reduce the noise during the evaluation we split the implementation into two different FPGAs: a control FPGA handles I/O with the host computer and supplies data in masked representation to the crypto FPGA. Crypto FPGA holds a PRNG, which generates the randomness for refreshing, and our masked KECCAK designs. In order to get the cleanest possible traces, we clock the PRNG in the negative edge to avoid extra noise and use a very slow $3 \mathrm{MHz}$ clock so that we are sure there is no overlap in the power consumption between cycles. The design is synthesized using the Xilinx tools with the property KEEP HIERARCHY set to yes, in order to avoid optimizations among the different blocks what would compromise the security of the design. We sample at $1.0 \mathrm{GS} / \mathrm{s}$ with 2000 points per frame over two rounds and a half.

TVLA. We use non-specific test vector leakage assessment (TVLA) method described in $\left[\mathrm{CMG}^{+} 13\right]$ to detect leakage. Note that this t-test based leakage detection method is not used to mount an attack and retrieve the key. From a theoretical point of view, the presence of leakage is a necessary, but not sufficient condition for an attack to succeed.

\footnotetext{
${ }^{1}$ Please note that we have contacted the authors of [GSM17a]. They have acknowledged our findings and provided an update of their implementation accordingly [GSM17b]
} 

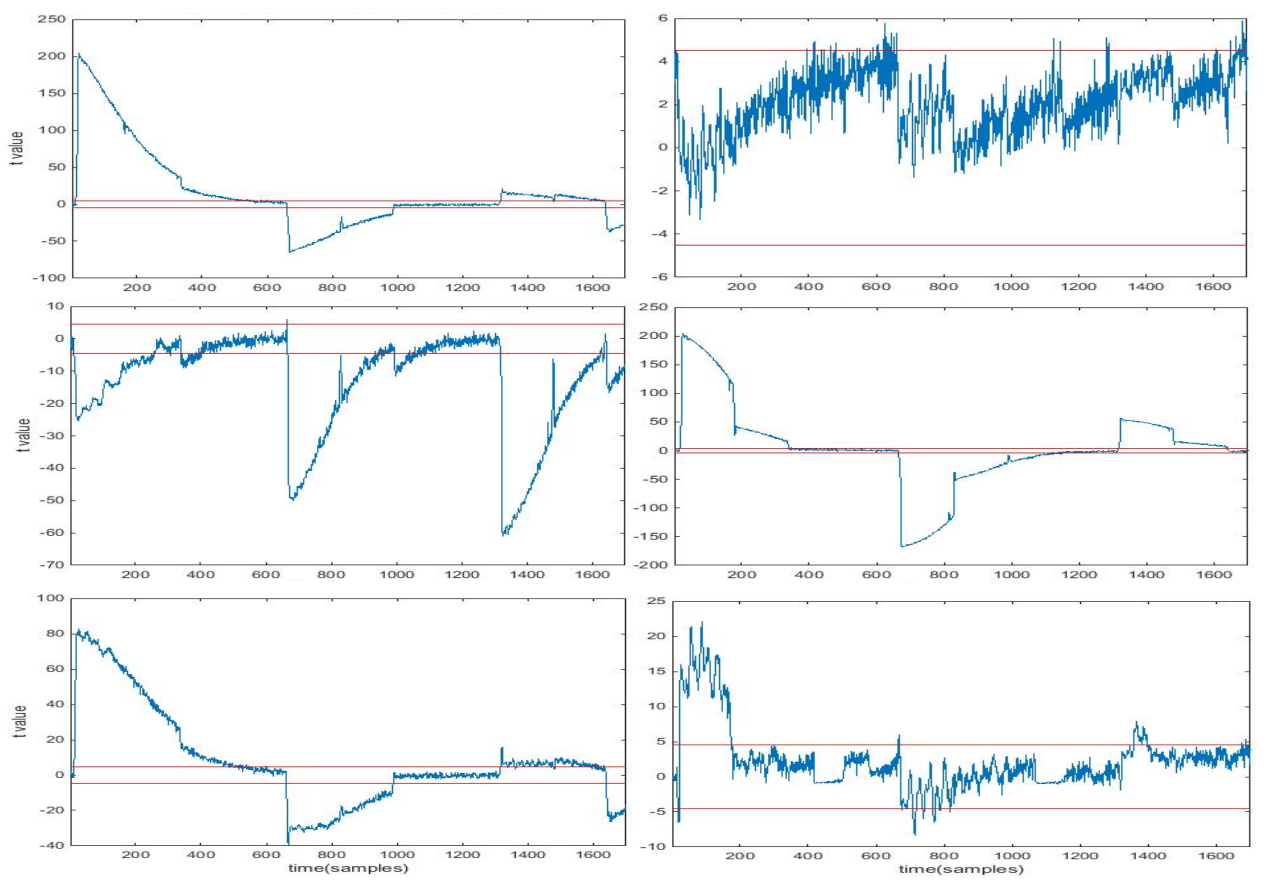

Figure 2: TVLA on our first-order DOM-KECCAK implementation. Left: Masks off with 20k measurements. Right: masks on with 55M measurements. From top to bottom: first-, second-, and third-order analysis respectively.

However, not observing leakage gives confidence to the designer. We choose our confidence level to be $99.9995 \%$ which corresponds to absolute t-values being greater than 4.5 for failure.

Two different tests are performed, namely, Fix vs. Random with masks off and with masks on. In both cases the idea is to compare the power consumption of the KECCAK- $f$ given to different states, the first one, chosen at random and fixed through all the encryptions and the second one, randomly chosen as well, that changes for every encryption. The test determines whether it is possible to distinguish between one another and hence, find potentially exploitable leakage depending on the state. When masks are off leakage is expected, since the countermeasures are switched off. On the other hand, when masks are on we expect no first-order leakage for a first-order implementation. We ensure that the initial sharing is done properly and focus our analysis only on the first two rounds of the cipher to validate our claims. All designs are analyzed under this same conditions in this work.

Figure 2 shows results of TVLA for 20 thousand traces when masks are off and 55 million traces when masks are on for a DOM-KECCAK implementation. In this case, although the leakage is reduced greatly, we see that the t-score goes over 4.5 for a significant number of sample points during both first and second rounds. We emphesize that we make no claims on this observation leading to a successful attack. Our goal is to make leakage assessment comparison between this implementation and our proposal which is presented in the next section.

\subsection{Non-Complete Round-Based Architecture}

The question that naturally arises after this analysis is the root of the non-completeness failure. Clearly, it is important to take into account the effect of the linear layers in 


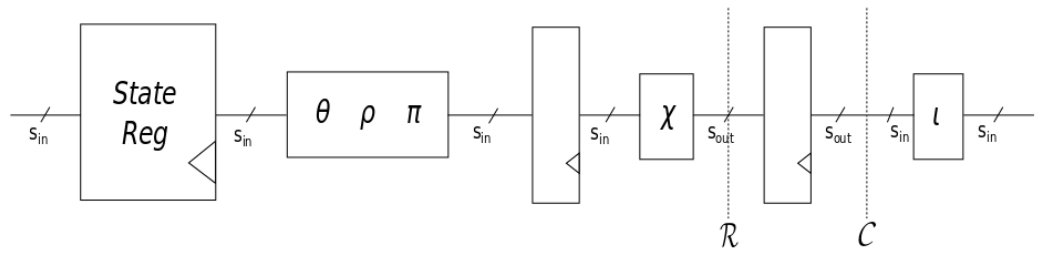

Figure 3: One round structure of the naive fix, with refreshing $(\mathcal{R})$ and compression $(\mathcal{C})$ before $\iota$.

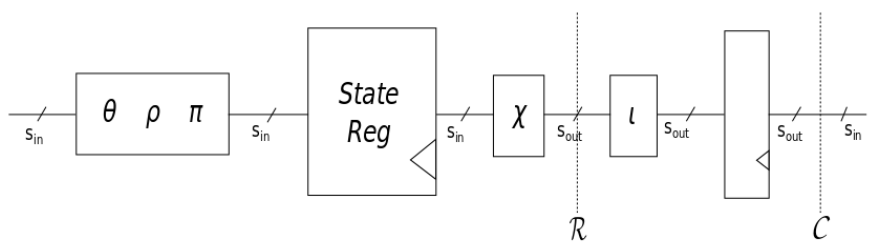

Figure 4: One round structure of NC-KECCAK, with the state register after the linear permutations and $\iota$ before the second layer of registers.

combination with the non-linear layer within a cycle, since $\chi$ and the linear operations including $\theta$ are secure individually. A detailed observation shows the following.

Order of operations. The order of linear and non-linear operations within a round is important. If the round structure of a cryptographic algorithm follows the traditional SPN approach where the linear operation follows the non-linear operation within a round, we would not observe this failure of non-completeness if the non-linear operation is noncomplete. For higher orders, one still needs to ensure composibility, which is not the objective of this paper's discussion.

Linear transformations. The structure of KECCAK linear operations, in particular $\theta$ which shuffles and combines several state bits, causes the non-completeness to fail when $\chi$ is applied later. Operations $\rho$ and $\pi$ being simple wirings do not combine several bits and hence do not cause any problem.

Naive fix. Following above observations, one can clearly see that it suffices to introduce a register between the linear operations and $\chi$ to secure the design. However, this new layer of registers increases the number of cycles required per round without a significant increase in maximum frequency. Fig. 3 illustrates this architecture. However, the main goal tried to achieve with a parallel implementation is a low-latency design, compared to a serial implementation that would have a higher latency but smaller area.

Our design. In order to reduce the latency, we push the state registers to after the linear operations, using this layer of registers to break the dependencies created by these operations. In this case we need two clock cycles to perform one round and hence 36 cycles for KECCAK- $f[200]$. Figure 4 presents this structure, with which the implementation is fulfilling non-completeness. Note that it is possible to neg-edge clock the second layer of registers saving one clock cycle and reducing the area by approximately 2800GE. Here, we use two separate registers for clear analysis of the target difference. 


\subsubsection{Evaluation}

We present the results for our non-complete KECCAK (NC-KECCAK) for both first- and second-order implementations, which follow the structure described in Fig. 4. We kept exactly the same setup as in Section 3.1.1 for comparability. In the case of the first-order secure implementation no first-order leakage appears, while in the second-order neither first- nor second-order leakage appear up to 55 million traces. Fig. 5 presents these results.
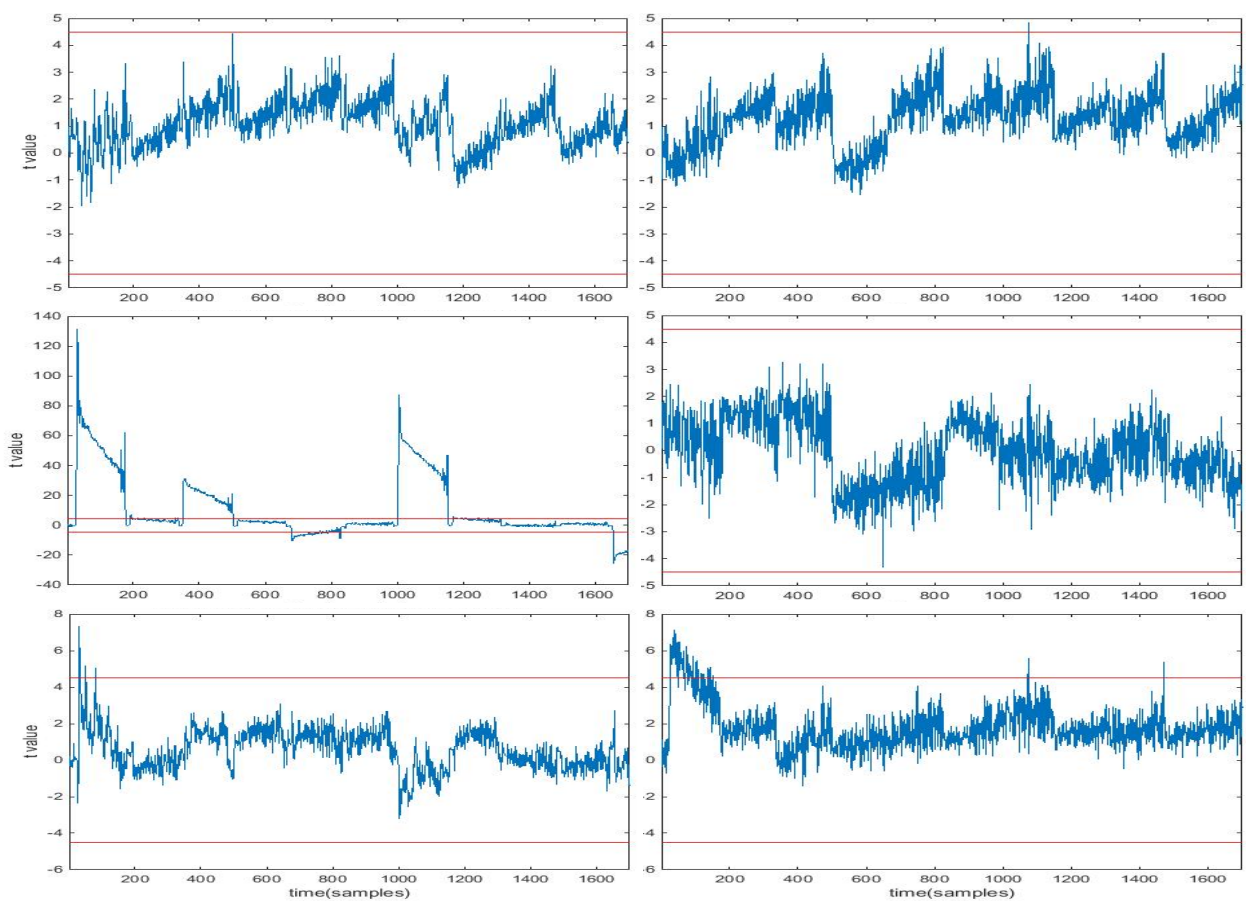

Figure 5: TVLA on our first (left) and second-order (right) NC-KECCAK implementations over 55M traces. From top to bottom: first-, second-, and third-order analysis respectively.

\subsection{Performance Analysis}

To synthesize our designs we use Synopsys Design Compiler Version I-2013.12 with NanGate 45nm Open Cell Library. The synthesis is done by using the -compile command and setting the flag -exact_map to avoid any optimization that could affect the security. The option -no_autoungroup is set by default when using -compile. This way it keeps the hierarchy of the design so that no optimizations in between modules can happen. Table 1 shows the results for this section's implementations. Note that the number of random bits presented is for each round, i.e., for every two clock cycles, and can be reduced significantly using the ideas from [BDN ${ }^{+} 14$, GSM17a, Dae17] which is not the goal of this paper. What is important is that the performance results with and without the suggested fix is comparable.

\section{Unrolled Implementations}

A typical way to gain low-latency is using an unrolled implementation where more than one round of the algorithm is implemented to finish within one cycle. Even though examples of such unprotected implementations exist, there are no such SCA protected implementations to our knowledge. 
Table 1: Synthesis results for the different designs

\begin{tabular}{|c|c|c|c|c|c|c|c|}
\hline \multirow[b]{2}{*}{ DESIGN } & \multicolumn{4}{|c|}{$\operatorname{AREA}(\mathrm{GE})$} & \multirow[b]{2}{*}{ Rand. } & \multirow[b]{2}{*}{ Cycles } & \multirow{2}{*}{$\begin{array}{c}\text { Max.Freq. } \\
(\mathrm{MHz})\end{array}$} \\
\hline & $\chi$ & $\theta$ & State & Total & & & \\
\hline Plain & 542.63 & 638.4 & 1333 & 2759 & - & 18 & 1136 \\
\hline DOM-KECCAK 1st & $9881^{*}$ & 1600 & 2667 & 17105 & 200 & 36 & 1087 \\
\hline NC-KECCAK 1st & 2613 & 1600 & $5334^{*}$ & 17493 & 200 & 36 & 1300 \\
\hline DOM-KECCAK 2nd & $23177^{*}$ & 2400 & 4000 & 33535 & 600 & 36 & 1111 \\
\hline NC-KECCAK 2nd & 6200 & 2400 & $12001^{*}$ & 35223 & 600 & 36 & 1205 \\
\hline
\end{tabular}

* This number includes the registers layer needed before the compression

In what follows, we discuss the input and output shares on a unrolled implementations and their impact on the choice of security level in each round/layer. In order to do that, we first focus on unrolling a quadratic function twice, i.e. implementing two layers in one cycle. Then we generalize our observation to implement $F=R^{N} \circ \cdots \circ R^{1}$ with $N$ layers, each layer being a degree $t_{R^{i}}$ function, in one cycle. We derive a formula of security order to consider in each layer $\left(d_{R^{i}}\right)$ without specifying the exact sharing or any claims on optimality.

\subsection{Quadratic Functions}

Let $R^{i}$ be a quadratic round function $\left(t_{R^{i}}=2\right)$, similar to that of KECCAK permutations. And let us initially target two rounds/layers, $F=R^{2} \circ R^{1}$. That is, we want to share and calculate not a quadratic, but a quartic function $\left(t_{F}=4\right)$ in a cycle.

First-order resistance. For additional simplicity, let's assume $R^{i}$ is composed of a series of AND gates. The dependency on the input bits for one output bit is provided in Fig. 6 . The indices in the parenthesis describe the input shares used in each share of a particular intermediate variable given TI as the underlying sharing. It is clear that if we use a first-order secure sharing for the first layer (Fig. 6, left), the first layer would indeed be secure. However, the second layer which uses the first layer's output can not satisfy non-completeness, i.e. every output share of $F$ would depend on all input shares of $F$.

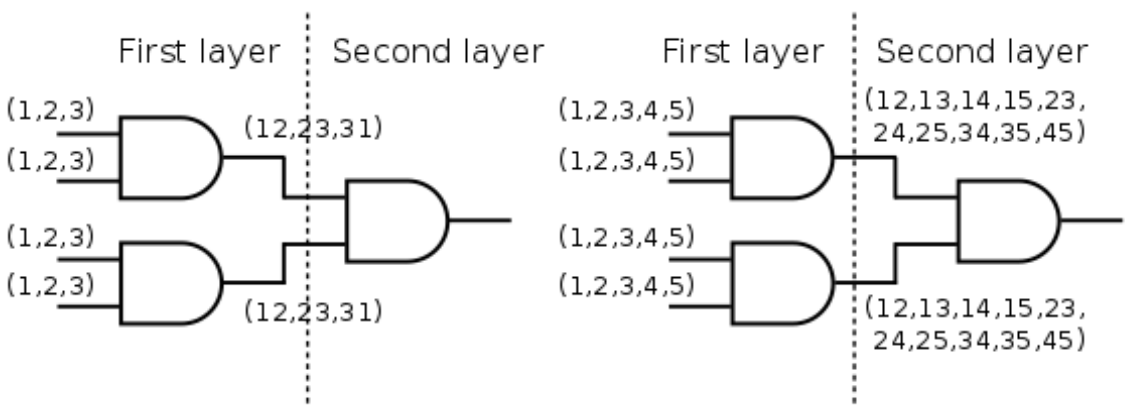

Figure 6: Quartic operation with (left) first-order sharing and (right) second-order sharing for the first round/layer of operations.

Since the security degrades with each nonlinear layer due to combination of shares, and that we aim first-order security even at the last layer, we decided to define the security order of each layer by crawling backwards from a single output bit to input bits and observing the requirements. Moreover, we benefit from the $d^{\text {th }}$-order non-completeness 
property. That is, we observe that first-order non-complete output bits of $F$ requires each combination of two input shares of $R^{2}$ being independent of the secret. That is any combination of two output shares of $R^{1}$ should be independent of the input which is the definition of second-order non-completeness. Hence, if we implement $R^{1}$ and $R^{2}$ such that they satisfy second- and first-order non-completeness respectively, $F$ would satisfy first-order non-completeness (Fig. 6, right).

Second-order resistance. We can repeat the same train of thought to analyze the security level each layer needs for second-order secure $F$. That is, if each combination of two output shares of $R^{2}$ is required to be independent of the secret, due to the nature of 2-input AND gate, each combination of four output shares of $R^{1}$ should be independent of the secret. This implies a fourth-order secure implementation for the first layer whereas a second-order secure implementation for the second layer.

Linear layer. It is only natural to question the impact of linear layers between quadratic operations. If we use a TI with $t d+1$ sharing instead of a $d+1$ DOM-indep like sharing, we have the freedom to have dependent shared inputs in each layer leading to a trivial inclusion of linear layers by simply producing the $j^{\text {th }}$ output shares using only $j^{\text {th }}$ input shares. We emphasize here the importance of considering the non-completeness of the whole cycle instead of only nonlinear layers.

\subsection{Extending the Methodology}

The above observation can be extended to higher degree functions and more rounds. Consider $F=R^{N} \circ \cdots \circ R^{1}$ where target security level for $F$ is $d_{F}$. Similar to Section 4.1, we can crawl back to decide the security level desired in each layer by using the following recursive formula:

$$
d_{R^{i}}= \begin{cases}d_{F} & \text { if } i=N, \\ t_{R^{i+1}} * d_{R^{i+1}} & \text { if } i<N\end{cases}
$$

Note that the above recursion is independent of the masking scheme. It neither specifies the particular sharing to be used in each layer for all possible functions nor necessarily provides the optimal result. Moreover, when higher-order SCA resistance is considered, the particularities of the refreshing layer becomes critical to provide multi-variate security. Clearly, the above formula does not specify the re-masking that should be used in order to provide multi-variate security for the whole algorithm. Hence, it should be taken as a suggested starting point rather than a complete recipe. One should always verify the security of the complete cycle and its composibility independently.

\section{Speeding Up Keccak Implementations}

Our goal in this section is to push the limits of low-latency masked KECCAK implementation using standard CMOS-like cells. Clearly, it is challenging to provide a secure unrolled implementation of KECCAK that calculates more than one round in one clock cycle using a $s_{i n}=d+1$ share masking scheme due to its high diffusion per round possibly causing dependencies in shared input variables. However, TI becomes advantageous in this setting with its increased number of shares and security even with dependent shares inputs.

We first discuss following Eqn. (6) where TI is used as the masking scheme and $s_{i n}$ is taken as the minimum from Eqn. (2). We elaborate on the challenges we had and the possibility of using another TI sharing with bigger $s_{i n}$ to reduce the cost and increase performance. Our security analysis and synthesis results indicate that this is the fastest protected hardware implementation published to this date. 


\subsection{First Attempt for Keccak}

According to Eqn. (2), a first-order SCA resistant implementation of a quartic function requires $s_{\text {in }} \geq 5$ shares given the algebraic normal form (ANF) of this function. However, for KECCAK and many other algorithms alike having a non-complete and uniform sharing based on the ANF of $F$ is not trivial due to the high diffusion of state bits. For such algorithms having a systematic approach such as the one described in Section 4 can be useful.

Sharing $\boldsymbol{R}^{\mathbf{1}}$. Following Eqn. 6 we start with a second-order non-complete TI sharing with $s_{\text {in }}=5$ and $s_{\text {out }}=10(5 \rightarrow 10)$ for $R^{1}$. We provide the output dependencies on the input shares for one such sharing in Eqn. (7). The exact sharing, which is detailed in App. A.4.1, is based on the sharing of an AND/XOR gate introduced in $\left[\mathrm{BGN}^{+} 14 \mathrm{a}\right]$. It can be verified that every combination of two outputs is independent of at least one input share.

$$
\begin{aligned}
S_{1}^{\prime} & =f_{1}\left(S_{1}, S_{2}\right) & S_{6}^{\prime} & =f_{6}\left(S_{1}, S_{3}\right) \\
S_{2}^{\prime} & =f_{2}\left(S_{2}, S_{3}\right) & S_{7}^{\prime} & =f_{7}\left(S_{3}, S_{5}\right) \\
S_{3}^{\prime} & =f_{3}\left(S_{3}, S_{4}\right) & S_{8}^{\prime} & =f_{8}\left(S_{5}, S_{2}\right) \\
S_{4}^{\prime} & =f_{4}\left(S_{4}, S_{5}\right) & S_{9}^{\prime} & =f_{9}\left(S_{2}, S_{4}\right) \\
S_{5}^{\prime} & =f_{5}\left(S_{5}, S_{1}\right) & S_{10}^{\prime} & =f_{10}\left(S_{4}, S_{1}\right)
\end{aligned}
$$

Sharing $\boldsymbol{R}^{2}$. When we choose the sharing of $R^{2}$ that makes it first-order secure, we also need to ensure that the non-completeness holds when $R^{1}$ and $R^{2}$ are combined. Due to the linear layer of KECCAK- $f$, the AND gates in $R^{2}$ depend on the outputs of more than two AND gates of $R^{1}$. Hence, not every first-order non-complete sharing of $R^{2}$ leads to a non-complete F. Moreover, even though many direct $10 \rightarrow 10$ sharings of $R^{2}$ satisfying the above restriction can be derived following a simple procedure, clearly we prefer a compatible first-order secure non-complete sharing for $R^{2}$ where $s_{\text {in }}=10$ and $s_{\text {out }}=5$ $(10 \rightarrow 5)$ in order to avoid further increase in number of shares. Hereon, we call a sharing where $s_{\text {in }}>s_{\text {out }}$ a compression sharing. Note other other examples of compression sharings can be found in literature $\left[\mathrm{BGN}^{+} 15, \mathrm{BGN}^{+} 14 \mathrm{~b}\right]$.

For a target of $10 \rightarrow 5$ compression sharing, we need to be careful since many shares are combined. Eqn. (8) illustrates the input-output dependency of one possible sharing for $\mathcal{R}^{2}$. We provide the exact sharing we use in App. A.4.2.

$$
\begin{array}{lll}
S_{1}^{\prime \prime}=f_{1}\left(S_{1}^{\prime}, S_{2}^{\prime}, S_{3}^{\prime}, S_{6}^{\prime}, S_{9}^{\prime}, S_{10}^{\prime}\right) & \text { (Missing input } \left.S_{5}\right) \\
S_{2}^{\prime \prime}=f_{2}\left(S_{1}^{\prime}, S_{2}^{\prime}, S_{5}^{\prime}, S_{6}^{\prime}, S_{7}^{\prime}, S_{8}^{\prime}\right) & \text { (Missing input } \left.S_{4}\right) \\
S_{3}^{\prime \prime}=f_{3}\left(S_{1}^{\prime}, S_{4}^{\prime}, S_{5}^{\prime}, S_{8}^{\prime}, S_{9}^{\prime}, S_{10}^{\prime}\right) & \text { (Missing input } \left.S_{3}\right) \\
S_{4}^{\prime \prime}=f_{4}\left(S_{3}^{\prime}, S_{4}^{\prime}, S_{5}^{\prime}, S_{6}^{\prime}, S_{7}^{\prime}, S_{10}^{\prime}\right) & \text { (Missing input } \left.S_{2}\right) \\
S_{5}^{\prime \prime}=f_{5}\left(S_{2}^{\prime}, S_{3}^{\prime}, S_{4}^{\prime}, S_{7}^{\prime}, S_{8}^{\prime}, S_{9}^{\prime}\right) & \text { (Missing input } \left.S_{1}\right)
\end{array}
$$

\subsection{Our Final Design}

\subsubsection{Optimized Sharings}

The greatest area contributor of a KECCAK parallel implementation, is the $\chi$ step. The number of gates in the non-linear operation scales with the number of input shares as follows: 
- Number of AND gates $=\left(\begin{array}{c}s_{i n} \\ 2\end{array}\right)+s_{i n}$

- Number of XOR gates $=\left(\begin{array}{c}s_{i n} \\ 2\end{array}\right)+s_{i n}$

- Number of NOT gates $=s_{\text {in }}$

The area overhead of $R^{2}$ caused by increasing the number of shares to 10 is significant. Therefore, we decided to look for another sharing for the first layer that produces less outputs. One possible option would be the $6 \rightarrow 7$ sharing proposed in $\left[\mathrm{BGN}^{+} 14 \mathrm{a}\right]$. On the other hand, by investigating further we found $6 \rightarrow 6$ sharings for both layers which provides first-order non-completeness when combined. The abstract sharings and the exact equations are provided in Eqn. (9), and in App. A.4.3 and A.4.4 respectively.

$$
\begin{array}{lll}
S_{1}^{\prime}=f_{1}\left(S_{1}, S_{2}, S_{3}\right) & S_{1}^{\prime \prime}=f_{1}\left(S_{4}^{\prime}, S_{5}^{\prime}, S_{6}^{\prime}\right) & \text { (Missing input } \left.S_{1}\right) \\
S_{2}^{\prime}=f_{2}\left(S_{1}, S_{4}, S_{5}\right) & S_{2}^{\prime \prime}=f_{2}\left(S_{2}^{\prime}, S_{3}^{\prime}, S_{5}^{\prime}\right) & \text { (Missing input } \left.S_{2}\right) \\
S_{3}^{\prime}=f_{3}\left(S_{1}, S_{4}, S_{6}\right) & S_{3}^{\prime \prime}=f_{3}\left(S_{2}^{\prime}, S_{3}^{\prime}, S_{4}^{\prime}\right) & \text { (Missing input } \left.S_{3}\right) \\
S_{4}^{\prime}=f_{4}\left(S_{2}, S_{5}, S_{6}\right) & S_{4}^{\prime \prime}=f_{4}\left(S_{1}^{\prime}, S_{4}^{\prime}, S_{5}^{\prime}\right) & \text { (Missing input } \left.S_{4}\right) \\
S_{5}^{\prime}=f_{5}\left(S_{3}, S_{5}, S_{6}\right) & S_{5}^{\prime \prime}=f_{5}\left(S_{1}^{\prime}, S_{3}^{\prime}, S_{6}^{\prime}\right) & \text { (Missing input } \left.S_{5}\right) \\
S_{6}^{\prime}=f_{6}\left(S_{2}, S_{3}, S_{4}\right) & S_{6}^{\prime \prime}=f_{6}\left(S_{1}^{\prime}, S_{2}^{\prime}, S_{6}^{\prime}\right) & \text { (Missing input } \left.S_{6}\right)
\end{array}
$$

With this sharing we are able to reduce the area considerably without failing noncompleteness.

\subsubsection{Performance Analysis}

We present the performance results for two round unrolled implementation using the sharings presented in Sections 5.1 and 5.2.1 in Table 2 for comparison.

The cost of $\chi^{1}$ and $\theta^{1}$ indicates that $R^{1}$ in $5 \rightarrow 10 \rightarrow 5$ sharing is a bit cheaper than the one using $6 \rightarrow 6 \rightarrow 6$. However, $\chi^{2}$ of $5 \rightarrow 10 \rightarrow 5$ is significantly more expensive due to the greater number of inputs as expected. In the $6 \rightarrow 6 \rightarrow 6$ scheme the state register is a bit bigger since there is one more share to store. All things considered, the area reduction of our design compared to a first attempt is clear.

\begin{tabular}{|c|c|c|c|c|c|c|c|c|}
\hline \multirow[b]{2}{*}{ DESIGN } & \multicolumn{6}{|c|}{$\operatorname{AREA}(\mathrm{kGE})$} & \multirow[b]{2}{*}{ Cycles } & \multirow{2}{*}{$\begin{array}{c}\text { Max.Freq } \\
(\mathrm{MHz})\end{array}$} \\
\hline & $\chi^{1}$ & $\chi^{2}$ & $\theta^{1}$ & $\theta^{2}$ & State & Total & & \\
\hline $5 \rightarrow 10 \rightarrow 5$ & 13.53 & 59.89 & 4.28 & 9.75 & 6.7 & 99.34 & 9 & 395.25 \\
\hline $6 \rightarrow 6 \rightarrow 6$ & 22.40 & 22.16 & 5.66 & 5.66 & 8.03 & 70.12 & 9 & 436.7 \\
\hline \multicolumn{9}{|c|}{ Previous work* } \\
\hline $3 \mathrm{sh}\left[\mathrm{BDN}^{+} 14\right]$ & - & - & - & - & 27.2 & 116.6 & 25 & 592 \\
\hline $4 \mathrm{sh}\left[\mathrm{BDN}^{+} 14\right]$ & - & - & - & - & 36.3 & 139.4 & 24 & 588 \\
\hline D.c. $[\mathrm{GSM} 17 \mathrm{~b}]^{* *}$ & - & - & - & - & 38.9 & 100.5 & 48 & 803.9 \\
\hline Pipel. [GSM17b]** & - & - & - & - & 36.8 & 111.8 & 72 & 837.5 \\
\hline
\end{tabular}

Given the results, it is possible to see that the time needed for a single encryption is $20.61 \mathrm{~ns}$ when synthesizing the design with the NanGate $45 \mathrm{~nm}$ library.

Table 2: KECCAK- $f$ [200] first-order secure unrolled implementations' performance results using NanGate 45nm library. Comparison with Parallel implementations from $\left[\mathrm{BDN}^{+} 14\right]$ and PARALLEL double clocked and PARALLEL pipelined from [GSM17b]

* Implementations of KECCAK- $f[1600]$

** Results gathered with library UMC 130nm 


\subsubsection{Evaluation}

We evaluate the optimized design using the $6 \rightarrow 6 \rightarrow 6$ sharing. The test is done under the conditions already presented in Section 3.1.1. Since this implementation is much faster than the previous ones, the analysis covers 10 rounds of the cipher.

The results are presented in Fig. 7 for masks off and masks on. It is possible to appreciate that the countermeasures indeed prevent leakage from appearing in the firstorder. Moreover, we observe no leakage in the second or in the third order t-test using 55 million measurements. We attribute this to the noise introduced by the large number of shares used. Therefore, we can affirm this is the fastest first-order SCA resistant KECCAK implementation published to this date.
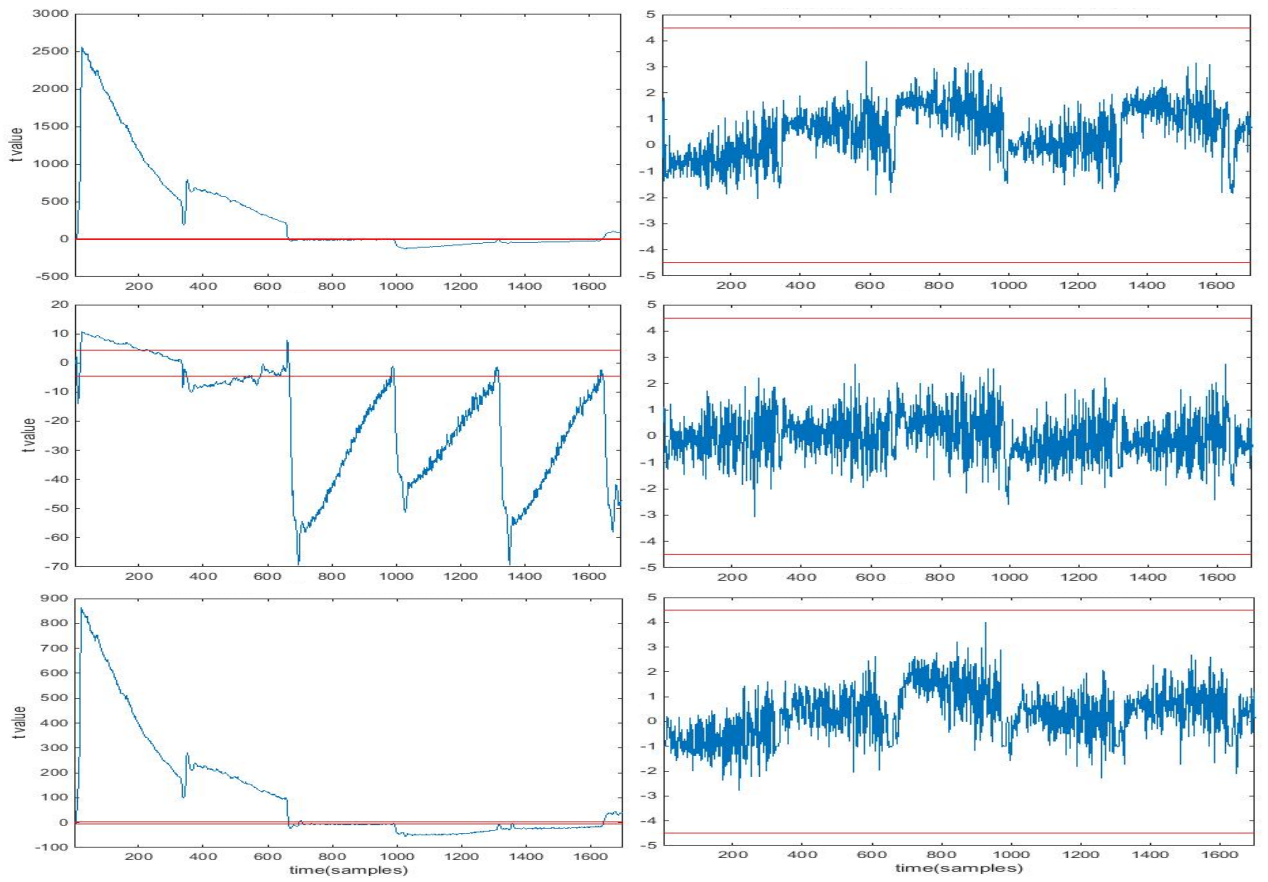

Figure 7: TVLA on first-order secure double round KECCAK implementation. Left: Masks off. Right: masks on. From top to bottom: first-, second- and third- order analysis.

\section{Conclusions}

In this work we presented, on the one hand, a flaw in previous round based KECCAK secure implementations on hardware and how to address this issue. We propose this solution for KECCAK permutations, but it is also applicable to other algorithms that concatenate several linear and non-linear operations. On the other hand, we introduced a method to speed up masked hardware implementations. Thus, by applying this method, the fastest SCA secure KECCAK implementation is presented.

\section{Acknowledgments}

This work was partially supported by COST Action (IC1306) through an STSM grant to George Petrides, by the Research Council KU Leuven (C16/15/058) and by the NIST Research Grant 60NANB15D346. Begül Bilgin is a Postdoctoral Fellow of the Fund for 
Scientific Research - Flanders (FWO). George Petrides is also affiliated to Eclat Enterprises Ltd, Cyprus.

\section{References}

$\left[\mathrm{BDN}^{+} 14\right]$ B. Bilgin, J. Daemen, V. Nikov, S. Nikova, V. Rijmen, and G. Van Assche. Efficient and first-order DPA resistant implementations of keccak. in CARDIS, volume 8419 of LNCS pp 187-199, June 2014.

$\left[\mathrm{BDP}^{+} 16 \mathrm{a}\right]$ G. Bertoni, J. Daemen, M. Peeters, G. Van Assche, and R. Van Keer. Caesar submission: Ketje v2, September 2016.

$\left[\mathrm{BDP}^{+} 16 \mathrm{~b}\right]$ G. Bertoni, J. Daemen, M. Peeters, G. Van Assche, and R. Van Keer. Caesar submission: Keyak v2, September 2016.

[BDPA10a] G. Bertoni, J. Daemen, M. Peeters, and G. Van Assche. Building power analysis resistant implementations of keccak. Second SHA-3 candidate conference, August 2010.

[BDPA10b] G. Bertoni, J. Daemen, M. Peeters, and G. Van Assche. The keccak reference. http://http://keccak.noekeon.org/, January 2010.

$\left[\mathrm{BGN}^{+} 14 \mathrm{a}\right]$ B. Bilgin, B. Gierlichs, S. Nikova, V. Nikov, and V. Rijmen. Higher-order threshold implementations. In ASIACRYPT, volume 8874 of LNCS, pages 326-343. Springer, 2014.

$\left[\mathrm{BGN}^{+} 14 \mathrm{~b}\right]$ B. Bilgin, B. Gierlichs, S. Nikova, V. Nikov, and V. Rijmen. A more efficient aes threshold implementation. In D. Pointcheval and Damien Vergnaud, editors, Progress in Cryptology-AFRICACRYPT 2014, volume 8469 of Lecture Notes in Computer Science, pages 267-284. Springer International Publishing, 2014.

$\left[\mathrm{BGN}^{+} 15\right]$ B. Bilgin, B. Gierlichs, S. Nikova, V. Nikov, and V. Rijmen. Trade-offs for threshold implementations illustrated on AES. Computer-Aided Design of Integrated Circuits and Systems, IEEE Transactions on, 34(7):1188-1200, July 2015.

$\left[\mathrm{BNN}^{+} 12\right] \quad$ B. Bilgin, S. Nikova, V. Nikov, V. Rijmen, and G. StÃijtz. Threshold implementations of all $3 \times 3$ and $4 \times 4$ s-boxes. In Emmanuel Prouff and Patrick Schaumont, editors, Cryptographic Hardware and Embedded SystemsCHES 2012, volume 7428 of Lecture Notes in Computer Science, pages 76-91. Springer Berlin Heidelberg, 2012.

$\left[\mathrm{BNN}^{+} 14\right] \quad$ B. Bilgin, S. Nikova, V. Nikov, V. Rijmen, N. Tokareva, and V. Vitkup. Threshold implementations of small s-boxes. Cryptography and Communications, pages 1-31, 2014.

[CBR $\left.{ }^{+} 15\right]$ T. De Cnudde, B. Bilgin, O. Reparaz, V. Nikov, and S. Nikova. Higher-order threshold implementation of the AES s-box. In Naofumi Homma and Marcel Medwed, editors, Smart Card Research and Advanced Applications - 14th International Conference, CARDIS 2015, Bochum, Germany, November 4-6, 2015. Revised Selected Papers, volume 9514 of Lecture Notes in Computer Science, pages 259-272. Springer, 2015. 
$\left[\mathrm{CMG}^{+} 13\right]$ J. Cooper, E. De Mulder, G. Goodwill, J. Jaffe, G. Kenworthy, and P. Rohatgi. Test vector leakage assessment (TVLA) methodology in practice. International Cryptographic Module Conference, 2013. http://icmc-2013.org/wp/ wp-content/uploads/2013/09/goodwillkenworthtestvector . pdf.

[Dae17] J. Daemen. Changing of the guards: A simple and efficient method for achieving uniformity in threshold sharing. In Wieland Fischer and Naofumi Homma, editors, Cryptographic Hardware and Embedded Systems - CHES 2017 - 19th International Conference, Taipei, Taiwan, September 25-28, 2017, Proceedings, volume 10529 of Lecture Notes in Computer Science, pages 137-153. Springer, 2017.

[DFS15] A. Duc, S. Faust, and F.-X. Standaert. Making Masking Security Proofs Concrete, pages 401-429. Springer Berlin Heidelberg, Berlin, Heidelberg, 2015 .

$\left[\right.$ FGP $\left.^{+} 17\right]$ S. Faust, V. Grosso, S. Merino Del Pozo, C. Paglialonga, and F.-X. Standaert. Composable masking schemes in the presence of physical defaults and the robust probing model. Cryptology ePrint Archive, Report 2017/711, 2017. http://eprint.iacr.org/2017/711.

[GMK16] H. Gross, S. Mangard, and T. Korak. Domain-oriented masking: Compact masked hardware implementations with arbitrary protection order. Cryptology ePrint Archive, Report 2016/486, 2016. http://eprint.iacr.org/2016/ 486.

[GMO01] K. Gandolfi, C. Mourtel, and F. Olivier. Electromagnetic analysis: Concrete results. In Çetin K. Koç, David Naccache, and Christof Paar, editors, Cryptographic Hardware and Embedded Systems-CHES 2001, volume 2162 of LNCS, pages 251-261. Springer Berlin Heidelberg, 2001.

[GSM17a] H. Gross, D. Schaffenrath, and S. Mangard. Higher-order side-channel protected implementations of keccak. In 2017 Euromicro Conference on Digital System Design (DSD), pages 205-212, Aug 2017.

[GSM17b] H. Gross, D. Schaffenrath, and S. Mangard. Higher-order side-channel protected implementations of keccak. Cryptology ePrint Archive, Report 2017/395, 2017. https://eprint.iacr.org/2017/395.

[ISW03] Y. Ishai, A. Sahai, and D. Wagner. Private Circuits: Securing Hardware against Probing Attacks, pages 463-481. Springer Berlin Heidelberg, Berlin, Heidelberg, 2003.

[KJJ99] P. C. Kocher, J. Jaffe, and B. Jun. Differential power analysis. In Advances in Cryptology - CRYPTO '99, 19th Annual International Cryptology Conference, Santa Barbara, California, USA, August 15-19, 1999, Proceedings, pages 388-397, 1999.

[NIS15] NIST. SHA-3 Standard: Permutation-based Hash and Extendable Output Functions. 2015.

[NRR06] S. Nikova, C. Rechberger, and V. Rijmen. Threshold implementations against side-channel attacks and glitches. In ICICS, volume 4307 of LNCS, pages 529-545. Springer, 2006.

[NRS08] S. Nikova, V. Rijmen, and M. Schlaffer. Secure hardware implementation of non-linear functions in the presence of glitches. In ICISC, volume 5461 of LNCS, pages 218-234. Springer, 2008. 
[NRS11] S. Nikova, V. Rijmen, and M. Schlaffer. Secure hardware implementation of non-linear functions in the presence of glitches. J. Cryptology, 24(2):292-321, 2011.

$\left[\mathrm{RBN}^{+} 15\right]$ O. Reparaz, B. Bilgin, S. Nikova, B. Gierlichs, and I. Verbauwhede. Consolidating Masking Schemes, pages 764-783. Springer Berlin Heidelberg, Berlin, Heidelberg, 2015.

[Sch17] D. Schaffenrath. Dom protected hardware implementations of keccak. https: //github.com/hgrosz/keccak_dom, 2017. 


\section{A Appendix}

\section{A.1 Keccak Round Steps}
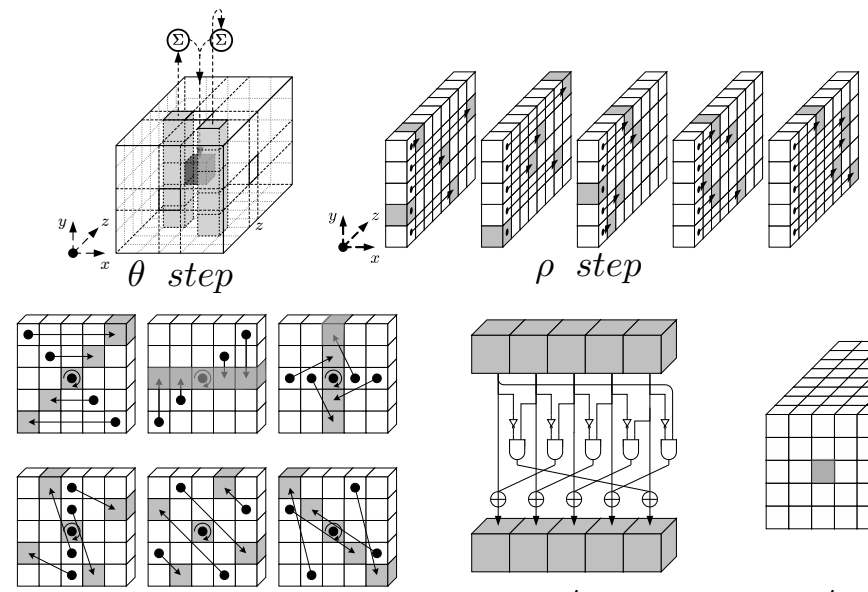

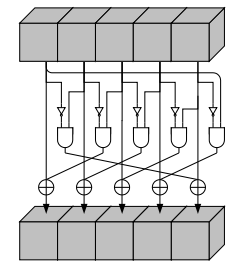

$\chi$ step

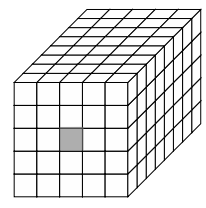

$\iota$ step

Figure 8: KECCAK- $f[200]$ state and steps [BDPA10b].

\section{A.2 Second-order Masking}

\section{A.2.1 Threshold Implementation ( $\mathrm{TI})$}

$$
\begin{aligned}
& z_{1}=a_{1} \oplus b_{1} c_{1} \oplus b_{1} c_{5} \oplus b_{5} c_{1} \\
& z_{3}=a_{3} \oplus b_{3} c_{3} \oplus b_{1} c_{3} \oplus b_{3} c_{1} \\
& z_{5}=a_{5} \oplus b_{5} c_{5} \oplus b_{2} c_{5} \oplus b_{5} c_{2} \\
& z_{7}=b_{2} c_{4} \oplus b_{4} c_{2} \\
& z_{9}=b_{3} c_{5} \oplus b_{5} c_{3}
\end{aligned}
$$

$$
\begin{aligned}
z_{2} & =a_{2} \oplus b_{2} c_{2} \oplus b_{1} c_{2} \oplus b_{2} c_{1} \\
z_{4} & =a_{4} \oplus b_{4} c_{4} \oplus b_{1} c_{4} \oplus b_{4} c_{1} \\
z_{6} & =b_{2} c_{3} \oplus b_{3} c_{2} \\
z_{8} & =b_{3} c_{4} \oplus b_{4} c_{3} \\
z_{10} & =b_{4} c_{5} \oplus b_{5} c_{4}
\end{aligned}
$$

\section{A.2.2 Domain Oriented Masking (DOM)}

$$
\begin{aligned}
& z_{1}=\left(a_{1} b_{1}\right) \oplus\left[a_{1} b_{2} \oplus r_{1}\right] \oplus\left[a_{1} b_{3} \oplus r_{2}\right], \\
& z_{2}=\left[a_{2} b_{1} \oplus r_{1}\right] \oplus\left(a_{2} b_{2}\right) \oplus\left[a_{2} b_{3} \oplus r_{3}\right], \\
& z_{3}=\left[a_{3} b_{1} \oplus r_{2}\right] \oplus\left[a_{3} b_{2} \oplus r_{3}\right] \oplus\left(a_{3} b_{3}\right) .
\end{aligned}
$$

\section{A.3 Non-completeness Failure in Round-Based DOM Implementation}

\section{A.3.1 First Order.}

Fig. 10 presents graphically what is demonstrated in Eqn.6 numerically:

\section{A.3.2 Second Order.}

Here we trace back two of the bits that together fail non-completeness in the second-order implementation, following the same idea as for the first-order case: 

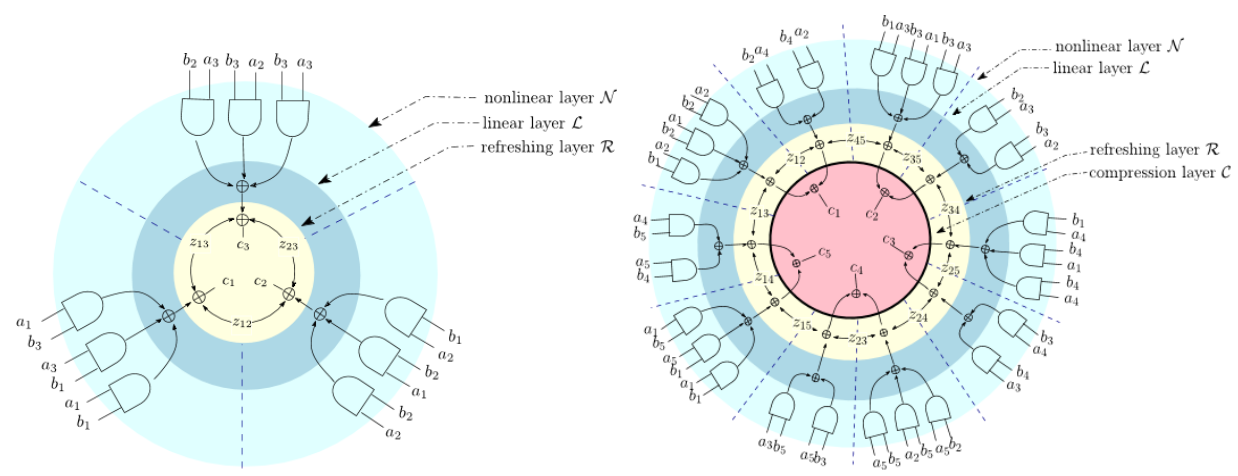

Figure 9: First- and second-order threshold implementation with randomness $\left[\mathrm{RBN}^{+} 15\right]$.

\section{First bit}

$$
\begin{array}{rll}
\chi^{-1}: \quad S_{2}^{\chi}[0,0,7] & \rightarrow S_{2}^{\pi}[0,0,7] \oplus S_{2}^{\pi}[1,0,7] S_{2}^{\pi}[2,0,7] \\
\pi^{-1}: \quad S_{2}^{\pi}[0,0,7] & \rightarrow S_{2}^{\rho}[0,0,7] \\
S_{2}^{\pi}[1,0,7] & \rightarrow S_{2}^{\rho}[1,1,7] \\
S_{2}^{\pi}[2,0,7] & \rightarrow S_{2}^{\rho}[2,2,7] \\
\rho^{-1}: \quad S_{2}^{\rho}[0,0,7] & \rightarrow S_{2}^{\theta}[0,0,7] \\
S_{2}^{\rho}[1,1,7] & \rightarrow S_{2}^{\theta}[1,1,3] \\
S_{2}^{\rho}[2,2,7] & \rightarrow S_{2}^{\theta}[2,2,4] \\
\theta_{1}^{-1}: \quad S_{2}^{\theta}[0,0,7] & \rightarrow S_{2}[0,0,7] \oplus \bigoplus_{y^{\prime}=0}^{4} S_{2}\left[4, y^{\prime}, 7\right] \oplus \bigoplus_{y^{\prime}=0}^{4} S_{2}\left[1, y^{\prime}, 6\right] \\
& S_{2}^{\theta}[1,1,3] & \rightarrow S_{2}[1,1,3] \oplus \bigoplus_{y^{\prime}=0}^{4} S_{2}\left[0, y^{\prime}, 3\right] \oplus \sum_{y^{\prime}=0}^{4} S_{2}\left[2, y^{\prime}, 2\right] \\
& S_{2}^{\theta}[2,2,4] & \rightarrow S_{2}[2,2,4] \oplus \bigoplus_{y^{\prime}=0}^{4} S_{2}\left[1, y^{\prime}, 4\right] \oplus \sum_{y^{\prime}=0}^{4} S_{2}\left[3, y^{\prime}, 3\right]
\end{array}
$$



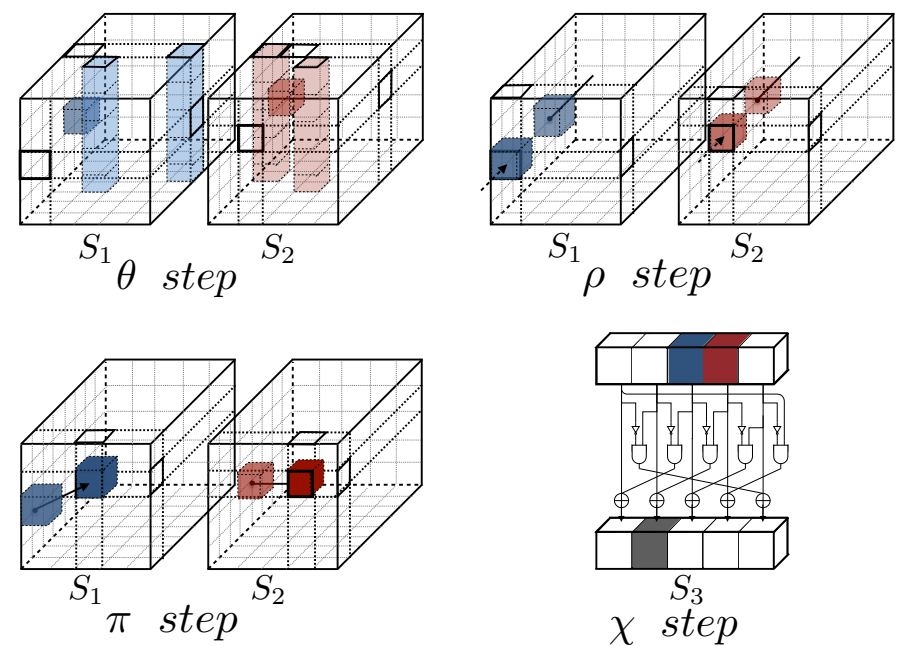

Figure 10: Tracing back output bit $C^{\chi}[4,1,0]$.

\section{Second bit}

$$
\begin{array}{rll}
\chi^{-1}: \quad S_{6}^{\chi}[2,0,5] & \rightarrow S_{1}^{\pi}[4,0,5] S_{3}^{\pi}[3,0,5] \\
\pi^{-1}: \quad S_{1}^{\pi}[4,0,5] & \rightarrow S_{1}^{\rho}[4,4,5] \\
S_{3}^{\pi}[3,0,5] & \rightarrow S_{3}^{\rho}[3,3,5] \\
\rho^{-1}: \quad S_{1}^{\rho}[4,4,5] & \rightarrow S_{1}^{\theta}[4,4,7] \\
S_{3}^{\rho}[3,3,5] & \rightarrow S_{3}^{\theta}[3,3,0] \\
\theta_{1}^{-1}: \quad S_{1}^{\theta}[4,4,7] & \rightarrow S_{1}[4,4,7] \oplus \bigoplus_{y^{\prime}=0}^{4} S_{1}\left[3, y^{\prime}, 7\right] \oplus \bigoplus_{y^{\prime}=0}^{4} S_{1}\left[0, y^{\prime}, 6\right] \\
& & \rightarrow S_{3}[3,3,0] \oplus \bigoplus_{y^{\prime}=0}^{4} S_{3}\left[2, y^{\prime}, 0\right] \oplus \sum_{y^{\prime}=0}^{4} S_{3}\left[4, y^{\prime}, 7\right]
\end{array}
$$




\section{A.4 Double round sharings}

\section{A.4.1 $5 \rightarrow 10$}

$\begin{aligned} S_{1}^{\prime}[x] & \leftarrow S_{1}[x] \oplus\left(S_{1}[x+1] \oplus 1\right) S_{1}[x+2] \oplus S_{1}[x+1] S_{2}[x+2] \oplus S_{2}[x+1] S_{1}[x+2] \\ S_{2}^{\prime}[x] & \leftarrow S_{2}[x] \oplus\left(S_{2}[x+1] \oplus 1\right) S_{2}[x+2] \oplus S_{2}[x+1] S_{3}[x+2] \oplus S_{3}[x+1] S_{2}[x+2] \\ S_{3}^{\prime}[x] & \leftarrow S_{3}[x] \oplus\left(S_{3}[x+1] \oplus 1\right) S_{3}[x+2] \oplus S_{3}[x+1] S_{4}[x+2] \oplus S_{4}[x+1] S_{3}[x+2] \\ S_{4}^{\prime}[x] & \leftarrow S_{4}[x] \oplus\left(S_{4}[x+1] \oplus 1\right) S_{4}[x+2] \oplus S_{4}[x+1] S_{5}[x+2] \oplus S_{5}[x+1] S_{4}[x+2] \\ S_{5}^{\prime}[x] & \leftarrow S_{5}[x] \oplus\left(S_{5}[x+1] \oplus 1\right) S_{5}[x+2] \oplus S_{5}[x+1] S_{1}[x+2] \oplus S_{1}[x+1] S_{5}[x+2] \\ S_{6}^{\prime}[x] & \leftarrow S_{1}[x+1] S_{3}[x+2] \oplus S_{3}[x+1] S_{1}[x+2] \\ S_{7}^{\prime}[x] & \leftarrow S_{3}[x+1] S_{5}[x+2] \oplus S_{5}[x+1] S_{3}[x+2] \\ S_{8}^{\prime}[x] & \leftarrow S_{5}[x+1] S_{2}[x+2] \oplus S_{2}[x+1] S_{5}[x+2] \\ S_{9}^{\prime}[x] & \leftarrow S_{2}[x+1] S_{4}[x+2] \oplus S_{4}[x+1] S_{2}[x+2] \\ S_{10}^{\prime}[x] & \leftarrow S_{4}[x+1] S_{1}[x+2] \oplus S_{1}[x+1] S_{4}[x+2]\end{aligned}$

A.4.2 $10 \rightarrow 5$

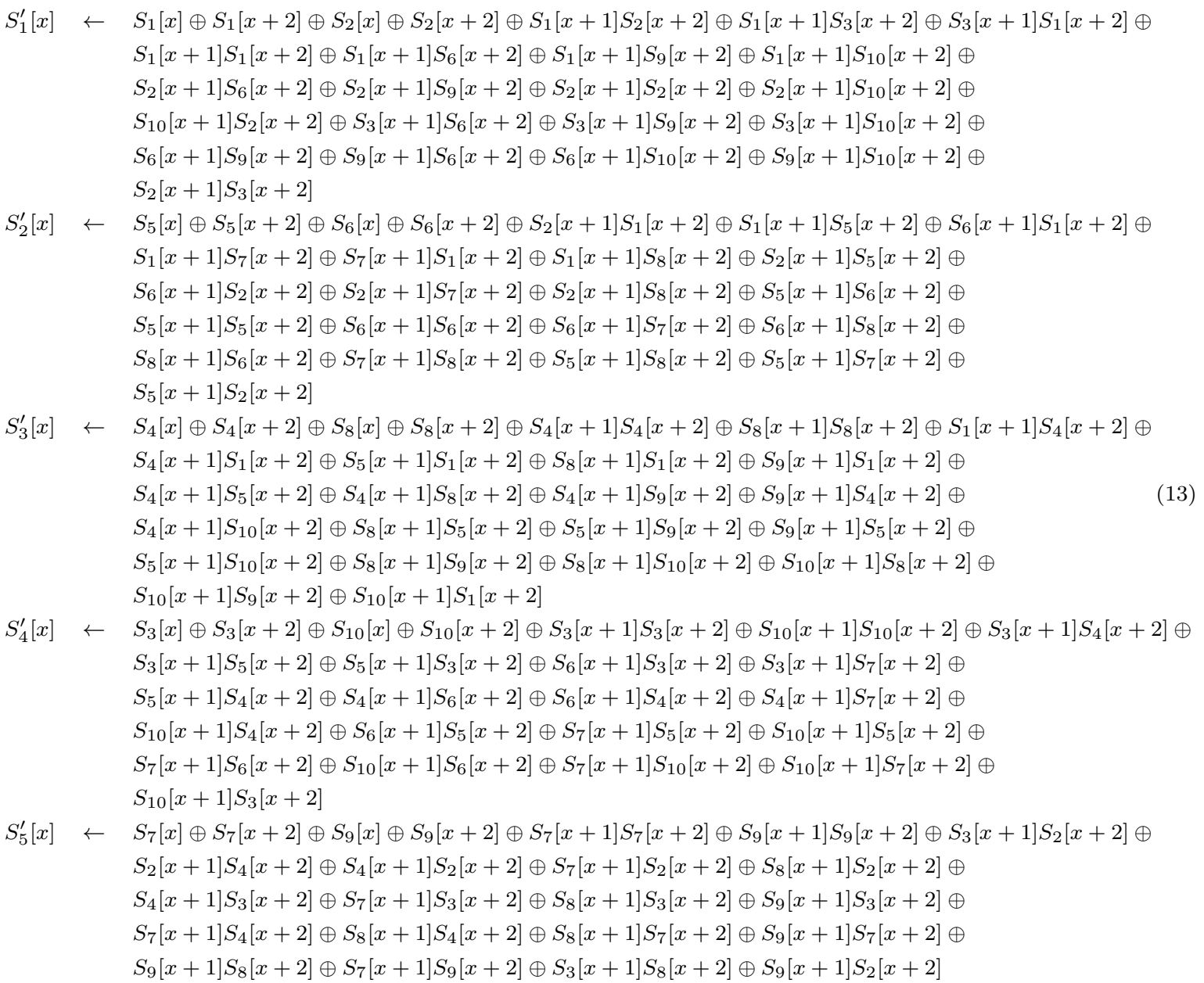




\section{A.4.3 First layer $6 \rightarrow 6$}

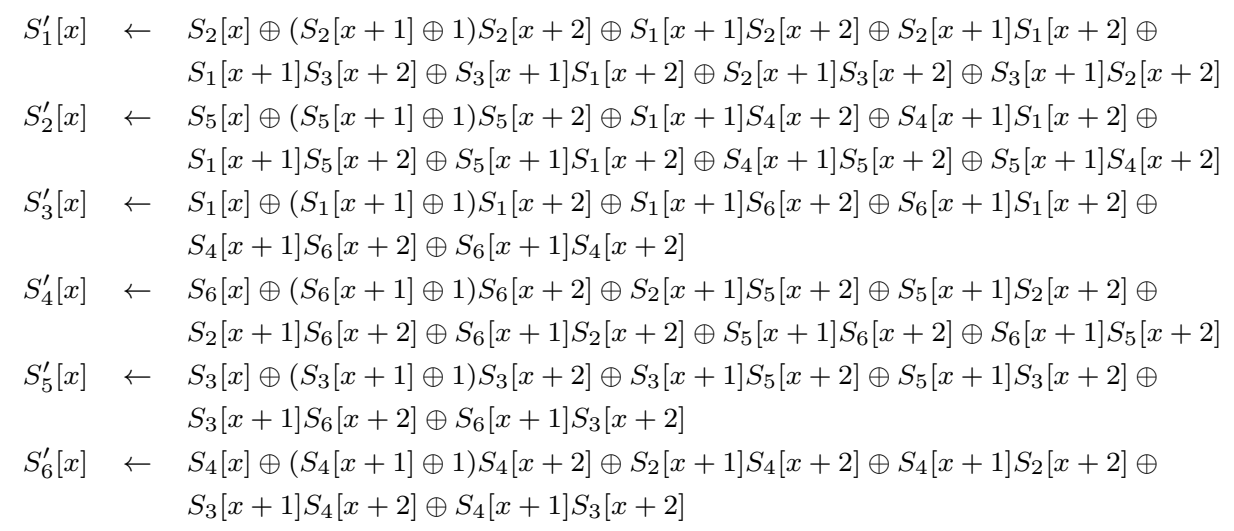

\section{A.4.4 Second layer $6 \rightarrow 6$}

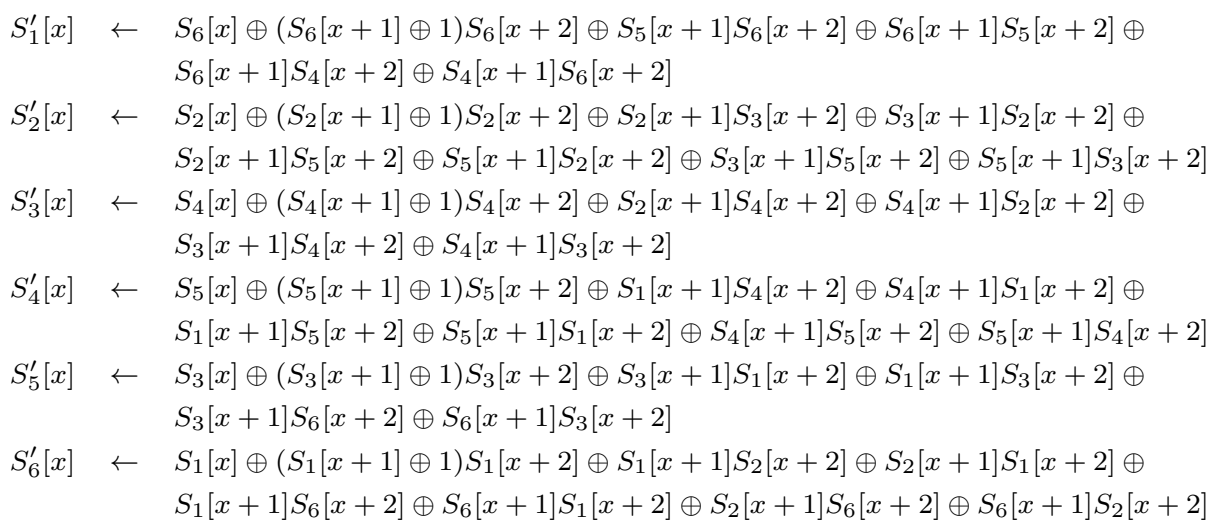

